\title{
Benchmarking the Application of Detached Eddy Simulation Techniques in Data Center Server Flow Modelling using Stereoscopic Particle Image Velocimetry
}

\author{
Sajad Alimohammadi \\ Technological University Dublin, sajad.alimohammadi@tudublin.ie \\ Jaakko McEvody \\ Trinity College Dublin, Ireland, jmcevoy@tcd.ie \\ Yan Delauré \\ Dublin City University, yan.delaure@dcu.ie
}

See next page for additional authors

Follow this and additional works at: https://arrow.tudublin.ie/engschmanconn

Part of the Mechanical Engineering Commons

\section{Recommended Citation}

Alimohammadi, S. et al. (2018) Benchmarking the Application of Detached Eddy Simulation Techniques in Data Center Server Flow Modelling using Stereoscopic Particle Image Velocimetry, 24th INTERNATIONAL WORKSHOP on Thermal Investigations of ICs and Systems (THERMINIC), IEEESeptember 2018, Stockholm.

This Conference Paper is brought to you for free and open access by the School of Manufacturing and Design Engineering at ARROW@TU Dublin. It has been accepted for inclusion in Conference Papers by an authorized administrator of ARROW@TU Dublin. For more information, please contact arrow.admin@tudublin.ie, aisling.coyne@tudublin.ie,gerard.connolly@tudublin.ie. Funder: Irish Research Council (IRC), SFI

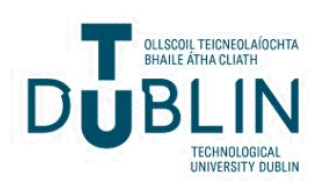




\section{Authors}

Sajad Alimohammadi, Jaakko McEvody, Yan Delauré, and Tim Persoons

This conference paper is available at ARROW@TU Dublin: https://arrow.tudublin.ie/engschmanconn/59 


\title{
Benchmarking the Application of Detached Eddy Simulation Techniques in Data Center Server Flow Modelling using Stereoscopic Particle Image Velocimetry
}

\author{
Sajad Alimohammadi ${ }^{1,2 *}$, Jaakko McEvoy ${ }^{1}$, Yan Delauré ${ }^{3}$, Tim Persoons ${ }^{1}$ \\ ${ }^{1}$ Department of Mechanical \& Manufacturing Engineering, the University of Dublin, Trinity College, TCD, Ireland \\ ${ }^{2}$ School of Mechanical \& Design Engineering, Dublin Institute of Technology, DIT, Ireland \\ ${ }^{3}$ School of Mechanical \& Manufacturing Engineering, Dublin City University, DCU, Ireland \\ * Corresponding Author: alimohas@tcd.ie, Phone: +35318961034
}

\begin{abstract}
The evolution and new trends in low-cost cloud computing services and growing data analysis loads on data centers keep increasing the power density of the servers as well as the global electricity consumption of data centers. The server component arrangement is mainly bounded by compactness of the device and can be somewhat overlooked. Both optical visualization and computational simulation techniques can be utilized to quantify complex turbulent flow fields inside the servers, and thus optimize thermal flows. The primary stage of a comprehensive research to enhance data center server cooling performance and energy efficiency is reported on here. A full-scale half-width $1 U$ blade server, i.e. a rectangular fanned channel, 150 mm by $40 \mathrm{~mm}$ by $500 \mathrm{~mm}$, air cooled by three parallel axial tube fans at the inlet is chosen as the base sever to be investigated using computational fluid dynamics CFD and stereoscopic particle image velocimetry PIV.
\end{abstract}

\section{Introduction}

Modern societies are experiencing an unprecedented growth of data centers with an increasing demand for cloud computing and data handling services. A growth of $15 \%$ in the data center industry was expected in the period from 2014 to 2018 [1]. With that comes the pressing rise in energy consumption for cooling to maintain the servers at ideal operating temperatures. This puts the emphasis on the need for an efficient and sustainable data center thermal management.

In the broad spectrum of data center thermal management research from the room level to the chip level, i.e. Macro to Nanoscale, a limited attention has been paid to utilizing advanced measurement and simulation techniques to visualize the very detailed description of full-scale air flows inside the server. This can be largely attributed to the complexity of the airflow inside the compact server which makes the validation of the results quite challenging.

Air cooled data center servers are yet the most demanding type whereas the liquid-cooled CPUs in hybrid-cooled servers are growing fast. For both cases of air- or hybridcooled servers, special consideration shall be given to component and board level design and developments.

As the densely packed racks are getting progressively more compact, the overall airflow distribution at the room level is no longer sufficient for the accurate prediction of the cooling at component level [2].

At the room or rack level, the airflow patterns within the various arrangements of the rack layouts have been extensively investigated to improve and optimize the load capacity and thermal efficiency [3]-[6]. This capacity to increase the thermal performance via server rack arrangement is being pushed to its limit while the overall processing power, i.e. the quantity of equipment and transistors in the dense integrated circuit, is increasing each year (Moore's Law).

Cooling at the individual board and chip level yet remains an open challenge for researchers. On-chip cooling is currently being largely investigated to deal with high fluxes as one of the most active fields of heat transfer [7]-[9]. Agostini et al. [7] have listed the main technologies currently used for onchip cooling as, single-phase flow in a microchannel, singleand two-phase flow in porous media, two-phase flow in a microchannel, and jet impingement cooling.

Regarding the server level flow visualization, Kumar and Joshi [10] performed planar PIV measurements to depict the air distribution across the rack inlet. They observed the effect of the flow rate variation on the air flow distribution at the server inlet leading to entrainment and reticulation effects for higher flow rates. Manoch et al. [11] have also considered 2D PIV combined with Reynolds Averaging CFD to map the flow field inside a rack module and have concluded that the SST RANS model is incapable of describing the flow field suitably.

This research pays special attention to the high fidelity airflow visualization inside the server using scale-resolving CFD and Stereoscopic PIV. The idea is to use a validated numerical-experimental analysis of air flow to develop new architectures for rack-mounted servers in the long run.

In the case of air-cooled servers using an array of rotary fans, a complex swirling flow is pushed into the inlet section of the server board which creates hugely turbulent features. This lies among the certain classes of flows that are not well covered using the well-known RANS turbulence models. As a result, special scale resolving simulation techniques combined with experimental validations are used to cope with the deficiencies in traditional models. 


\section{Methodology}

\subsection{Stereoscopic Particle Image Velocimetry (PIV)}

High-fidelity experimental measurements using Stereoscopic Particle Image Velocimetry (PIV) in the selected blade server channel are carried out. In a typical fan cooled data center server, the swirling flow emerging from the fans introduces a fully $3 \mathrm{D}$, non-symmetric, unsteady flow phenomena. The fans have an impeller diameter of $40 \mathrm{~mm}$, rated speed of $21,500 \mathrm{rpm}$ and maximum flow rate of $30 \mathrm{CFM}$. The crucial Stereo PIV validation experiments are carried out for the assessment of simulations. Two cameras from different angles view the plane capturing all three components of the velocity in the $2 \mathrm{D}$ plane. To preserve both view planes in focus, Scheimpflug lens arrangements are used.

Two CMOS cameras are used in a stereoscopic arrangement (LaVision High-Speed Star 6 at 500 fps with $1024 \times 1024$ px $^{2}$, 12 bit, and with Sigma $105 \mathrm{~mm} \mathrm{f} / 2.8$ EX DG Macro lenses). A glycol-water aerosol is used for seeding, with a particle diameter of 0.2-0.3 $\mu \mathrm{m}$. The beam of a Nd:YLF twin cavity laser (up to $15 \mathrm{~mJ}$ per pulse at $1 \mathrm{kHz}$ ) with the time step of $0.25 \mathrm{~ms}$ between two pulses passes through customized optics to form a $0.3 \mathrm{~mm}$ thick light sheet aligned to the mid-plane of the fan array channel, as shown in Fig. 1.

LaVision DaVis 7.2 software was used for image capturing and post-processing of the velocity fields, using multi-grid cross-correlation with continuous window shifting and deformation. A total number of image pairs for each camera was 2729 were used for average fields.

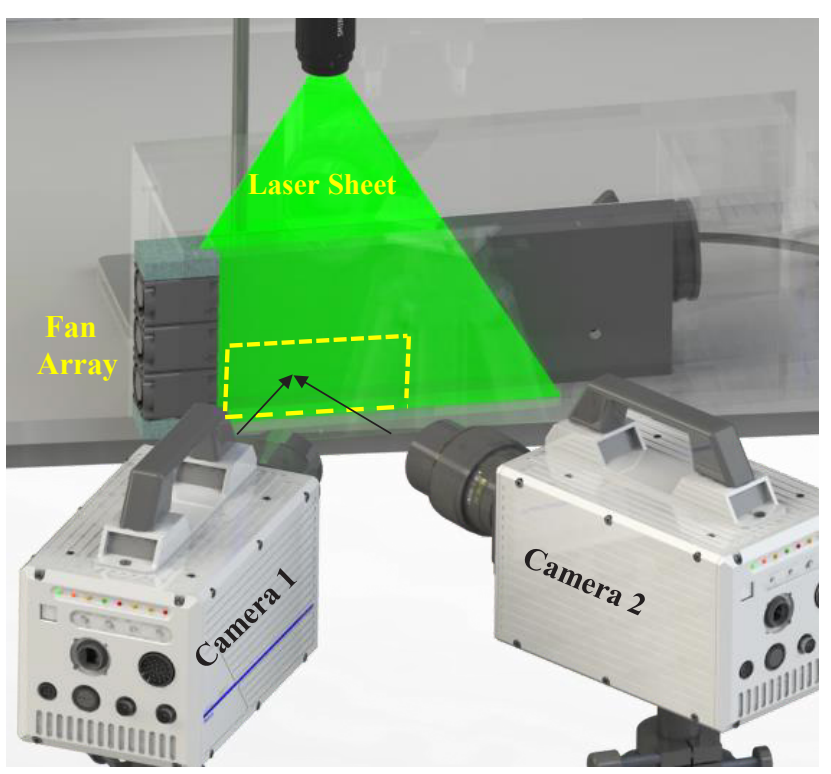

Figure 1: Stereo PIV Setup

\subsection{High Fidelity Simulations}

High-performance computational fluid dynamics using scaleresolving simulations (SRS) of the air flow, in which, at least parts of the turbulence spectrum are resolved in the numerical simulation. In hybrid RANS-LES models, turbulent features (eddies) at scales larger than the grid are resolved using Large Eddy Simulation LES, while in the vicinity of the wall where the turbulence length scale becomes very small, the region is modeled, i.e. not resolved, by RANS [12],[13]. This greatly reduces the mesh cell count in wall region where LES would require near DNS resolution.

Latest improvements in hybrid models such as Detached Eddy Simulation (DES) methods, in particular, suggest that accurate results for realistic operating conditions are feasible but careful validation of the near-wall treatment of grids is critical for accurate predictions. Specifically, this is an effort to utilize Many-core CFD simulations on data center cloud servers using Open Source packages and benchmark the performance of a recently developed turbulence model called Improved Delayed DES. The IDDES incorporates shielding functions to avoid Grid Induced Separation due to the switch from the inner RANS to outer LES and also provides a new definition for the length scale. It combines Delayed DES (DDES) and wall modeled LES (WMLES) based on the grid resolution and the inflow turbulent content [14].

Transient, incompressible simulations were carried out using pimpleFoam solver with an adjustable time step to enforce a maximum Courant number of 1.1 outer correction, 2 pressure correction, and 1 nonorthogonal correctors were used in the simulations. The discretization schemes used for temporal, gradient, and divergence terms are $2^{\text {nd }}$ order backward differencing, $2^{\text {nd }}$ order central differencing, and $2^{\text {nd }}$ order bounded central differencing, respectively.

Figure 2 displays the computational domain and the crosssectional view of the generated mesh. The mesh is fully structured with 7.5 MIO hexahedral type cells.

The simulations were carried out via batch processing on an on-demand high-end computing facility composed of an SGI ICE-X system (320 nodes $\times 2 \times 12$ cores - 16GB RAM @ 2.4 $\mathrm{GHz}$ ). A total of 120 cores were used per simulation.

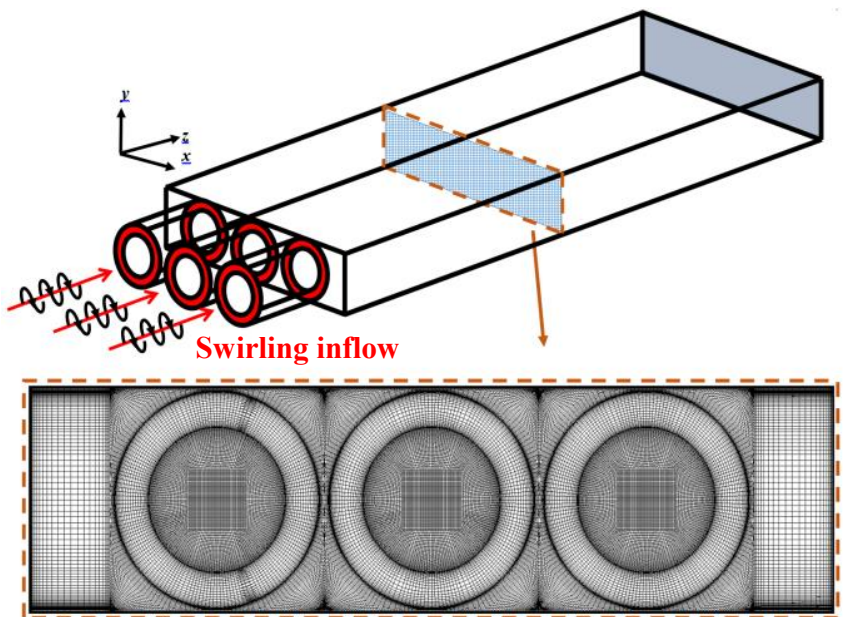

Figure 2: (a) CFD domain and with a view of mesh across the channel 


\section{Results}

The measurements and simulations are carried out for fans operating at $25 \%$ of their rated power $(25 \mathrm{~W})$ with a swirling inflow of 12 CFM, the rotational speed of 4895 RPM, and a blockage creating a back pressure of $0.055 \mathrm{kPa}$ to mimic the real server flow conditions.

\subsection{Validation}

The simulations results for the cross-wise distribution of the magnitude of flow velocity in comparison with Stereo PIV measurements are depicted in Fig. 3. CFD results are produced by both pure RANS, i.e. k- $\omega$ SST, and hybrid RANS-LES, i.e. IDDES, models based on a maximum Courant number of 1 .

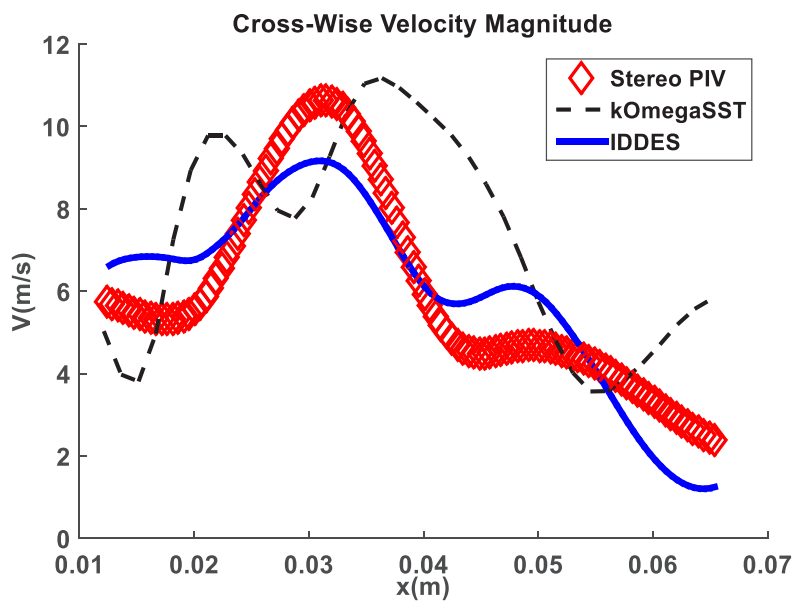

Figure 3: Distribution of the time-averaged cross-wise velocity (Stereo PIV vs CFD)

The RANS approach, dashed line, which models all turbulence length scales in the domain fails to predict the experiments. This is mainly due to the production of large turbulence levels in regions with strong accelerations and high anisotropy of turbulent stresses due to the swirling flow.

The hybrid simulation which resolves part of the turbulence spectrum, in comparison with the pure modeling approach, shows more promising results with a better approximation of mean flow velocity trends and peaks when compared to experiments. In terms of cross-wise velocity, the results show a relatively good agreement, while there are clear discrepancies when it comes to stream-wise velocity (not shown here). The maximum deviation of RANS results from the measurements is up to $55 \%$ while the hybrid method shows a better match with the maximum deviation of $24 \%$. It is worth noting that the IDDES method captures the trend of the PIV qualitatively well with two peaks in the velocity profile being aligned in the same locations for both measurements and simulations.

\subsection{Benchmarking}

IDDES combines some of the benefits of DDES and WMLES, as described earlier [14]. It shows a great potential to reproduce major experimentally observed features and to capture the swirling phenomena of the target industrial flow in a server with a better accuracy when compared to URANS. However, IDDES requires almost 4.5 times more processing time to accomplish the simulations. Additional improvements in the quantitative accuracy of the model can still be sought, i.e. through full LES mode, at the cost of multiplying the total computational time even further.

\subsection{Server Flows}

Figure 4 displays instantaneous scale resolving flow field simulations of velocity magnitudes drawn at the inlet section of the fanned channel, as a side by side comparison of URANS vs IDDES results. Figure 5 also compares the results for vorticity contours solved by two methods under analysis. It clarifies the level of the details that can be captured using hybrid RANS-LES methods.

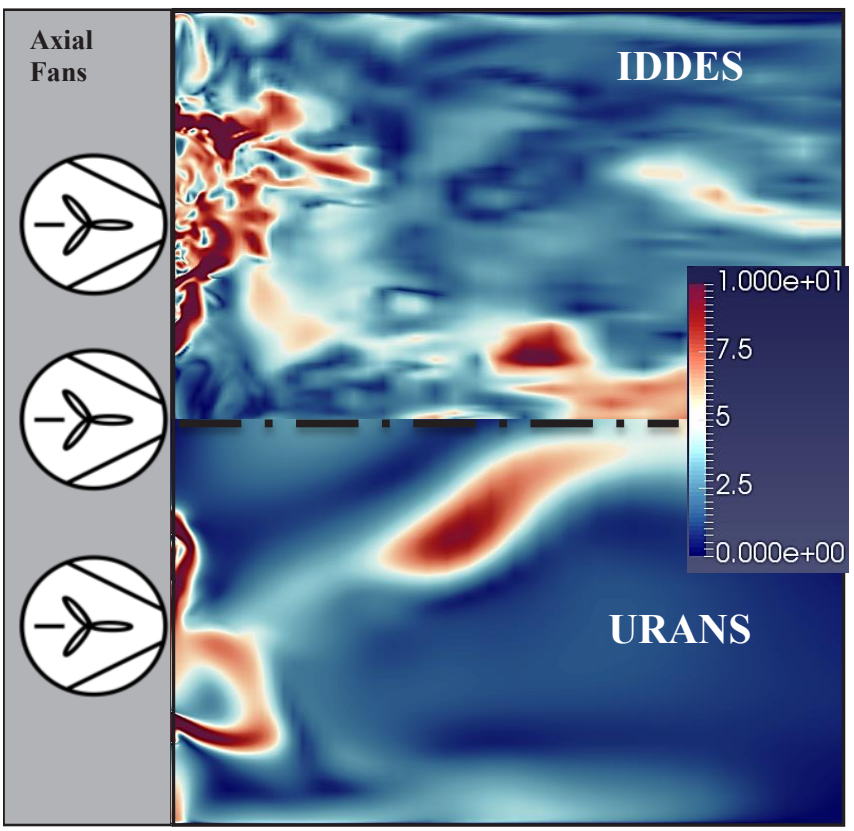

Figure 4: Comparison of velocity $(\mathrm{m} / \mathrm{s})$ flow fields by IDDES (top) vs URANS (bottom)

\section{Conclusions}

The research on data center thermal management at component and server level is considered as a key element in addressing the demand for novel technologies and to improve existing suboptimal air-cooled servers.

The applied research in hybrid methods for turbulent flows is relatively recent and to the authors' knowledge, no published study has addressed this type of electronic cooling problem for full-scale data center server flow simulations combined with Stereo PIV measurements. The excessive cost of Large Eddy Simulations for industrial applications, i.e. full-scale server flow simulations, can be reduced using several variants of hybrid methods which switch explicitly between LES and 


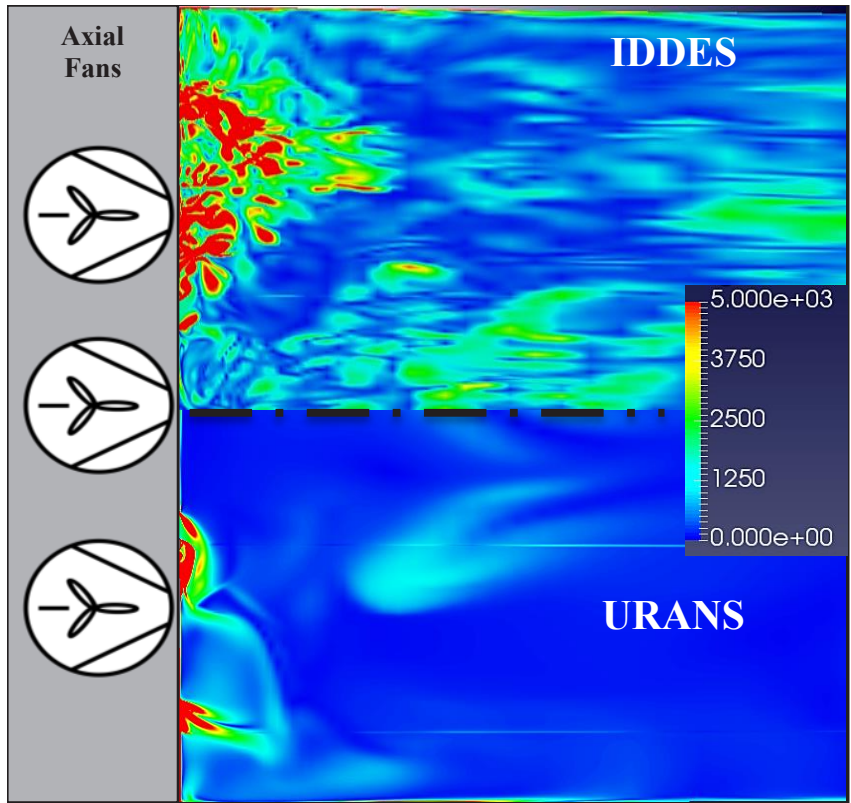

Figure 5: Comparison of vorticity (1/s) flow fields by IDDES (top) vs URANS (bottom)

RANS. IDDES, used here, is shown to better capture the trends in velocity profiles for the highly turbulent swirling flows under consideration. Results also show a marked improvement compared to the RANS.

The outcome of the first stage of this research results in the development of a modular experimental facility for air-cooled servers together with a computational modeling tool to efficiently simulate the air flow at the server level. This research has begun with side by side experimental-numerical comparison of a generic $1 \mathrm{U}$ server box and can be extended to include more comprehensive geometries and full-size racks composed of $\mathrm{nU}$ servers. The modeling strategy can help more frequent use of scale resolving simulations for the specific application of fan cooled data center servers where high fidelity measurements are not a feasible option. In the long term, this promotes the development of new design strategies for rack-mounted servers and improve the overall data center thermal management system with a primary focus on increasing the energy efficiency.

\section{Acknowledgments}

The authors also would like to acknowledge the support of the Irish Research Council (IRC) under grant number GOIPD/2016/216. This publication has partly emanated from research conducted with the financial support of Science Foundation Ireland under the SFI Strategic Partnership Programme Grant Number SFI/15/SPP/E3125. The authors also wish to acknowledge the DJEI/DES/SFI/HEA Irish Centre for High-End Computing (ICHEC) for the provision of computational facilities and support.

\section{Literature}

[1] S. V. Garimella, T. Persoons, J. A. Weibel, and V. Gektin, "Electronics Thermal Management in Information and Communications Technologies: Challenges and Future Directions", IEEE Trans. Compon. Packag. Manuf. Technol., vol. 7, no. 8, pp. 1191-1205, Aug. 2017.

[2] Y. Fulpagare and A. Bhargav, "Advances in data center thermal management”, Renew. Sustain. Energy Rev., vol. 43, pp. 981-996, Mar. 2015.

[3] S. A. Nada and K. E. Elfeky, "Experimental investigations of thermal managements solutions in data centers buildings for different arrangements of cold aisles containments", J. Build. Eng., vol. 5, pp. 41-49, Mar. 2016.

[4] S. Gondipalli, S. Bhopte, B. Sammakia, M. K. Iyengar, and R. Schmidt, "Effect of isolating cold aisles on rack inlet temperature", in 2008 11th Intersociety Conference on Thermal and Thermomechanical Phenomena in Electronic Systems, 2008, pp. 1247-1254.

[5] J. R. H. Schaadt, K. Fouladi, A. P. Wemhoff, and J. G. Pigeon, "Load Capacity and Thermal Efficiency Optimization of a Research Data Center Using Computational Modeling", 13th Int. Conf. Nanochan., Microchan., and Minichan., Jul. 2015.

[6] S. Alkharabsheh et al., "A Brief Overview of Recent Developments in Thermal Management in Data Centers", J. Electron. Packag., vol. 137, no. 4, pp. 040801-040801-19, Sep. 2015.

[7] B. Agostini, M. Fabbri, J. E. Park, L. Wojtan, J. R. Thome, and B. Michel, "State of the Art of High Heat Flux Cooling Technologies", Heat Transf. Eng., vol. 28, no. 4, pp. 258-281, Apr. 2007.

[8] J. B. Marcinichen, J. A. Olivier, and J. R. Thome, "Onchip two-phase cooling of datacenters: Cooling system and energy recovery evaluation", Appl. Therm. Eng., vol. 41, pp. 36-51, Aug. 2012.

[9] P. L. Leonard, and A.L. Phillips, "The Thermal Bus Opportunity - A Quantum Leap in Data Center", ASHRAE Trans.: Symp. Vol 111, pp. 732-745, 2005.

[10] P. Kumar and Y. Joshi, "Experimental investigations on the effect of perforated tile air jet velocity on server air distribution in a high density data center", 12th IEEE Inter. Conf. on Therm. and Thermomech. Phenomena in Electronic Systems, 2010, pp. 1-7.

[11] L. Manoch, J. Matěcha, and P. Pohan, "Flow field mapping in data rack model", EPJ Web Conf., vol. 45, p. 01061, 2013.

[12] Y. Hoarau, S.-H. Peng, D. Schwamborn, and A. Revell, Eds., "Progress in Hybrid RANS-LES Modelling: Papers Contributed to the 6th Symposium on Hybrid RANS-LES Methods", 26-28 September 2016, France.

[13] W. Haase, M. Braza, and A. Revell, Eds., "DESider - A European Effort on Hybrid RANS-LES Modelling", Springer, 2009.

[14] M. L. Shur, P. R. Spalart, M. K. Strelets, and A. K. Travin, "A hybrid RANS-LES approach with delayedDES and wall-modelled LES capabilities", Int. J. Heat Fluid Flow, vol. 29, no. 6, pp. 1638-1649, Dec. 2008. 